\title{
Self-Similar Solutions of the Kantowski-Sachs Model with a Perfect Fluid in General Relativity
}

\author{
Ragab M. Gad ${ }^{1,2}$, Sadah A. Alkhateeb' ${ }^{1}$, Hanan D. Alharbi ${ }^{1}$ \\ ${ }^{1}$ Mathematics Department, Faculty of Science, University of Jeddah, Jeddah, KSA \\ ${ }^{2}$ Mathematics Department, Faculty of Science, Minia University, El-Minia, Egypt \\ Email: ragab2gad@hotmail.com, salkhateeb@uj.edu.sa, hanan-510@hotmail.com
}

How to cite this paper: Gad, R.M., Alkhateeb, S.A. and Alharbi, H.D. (2021) SelfSimilar Solutions of the Kantowski-Sachs Model with a Perfect Fluid in General Relativity. Journal of Applied Mathematics and Physics, 9, 3165-3176.

https://doi.org/10.4236/jamp.2021.912207

Received: December 1, 2021

Accepted: December 25, 2021

Published: December 28, 2021

Copyright (c) 2021 by author(s) and Scientific Research Publishing Inc. This work is licensed under the Creative Commons Attribution International License (CC BY 4.0).

http://creativecommons.org/licenses/by/4.0/

\begin{abstract}
Exact self-similar solutions to Einstein's field equations for the Kantowski-Sachs space-time are determined. The self-similarity property is applied to determine the functional form of the unknown functions that define the gravitational model and to reduce the order of the field equations. The consequences of matter, described by the energy-momentum tensor, are investigated in the case of a perfect fluid. Some physical features and kinematical properties of the obtained model are studied.
\end{abstract}

\section{Keywords}

Kantowski-Sachs Model, Homothetic Vector Field, Einstein's Field

Equations, Kinematical Quantities

\section{Introduction}

Space-time symmetries are important in identifying features of space-time that exhibit some kind of symmetry. The most important symmetries are those that simplify Einstein's field equations and provide a space-time classification based on the corresponding Li-algebra configuration. These symmetries preserve certain physical properties of space-time, such as metric, geodesic, curvature, Ricci scalar, and energy-momentum tensor. In the context of the theory of General Relativity (GR), symmetries have been studied based on Riemannian geometry and on Lyra geometry and in the framework of the theory of teleparallel gravity based on Weitzenböck geometry. Various types of symmetries, such as isometry, homothetic, conformal, Ricci and matter collineations, etc, have been thoroughly studied in GR [1]-[18]. Some of these symmetries have also been examined in the theory of teleparallel gravity [19] [20]. In the context of exact self-similar solutions to Einstein's equations in GR, based on Lyra geometry, there are very few 
studies in the literature [21] [22] [23] [24].

Self-similar solutions to Einstein's field equations play an important role in describing asymptotic properties of more general solutions and are therefore of great interest in physics [25]. These solutions are relevant to astrophysics and critical phenomena in gravitational collapse, see the references [26] [27] [28] [29] [30].

Einstein's field equations of GR are a system of nonlinear partial differential equations in some independent variables, depending on the matter distribution, determined by the stress-energy tensor, and unknown scale factors. This complicated system cannot generally be integrated without making simplistic assumptions on the variables to get their exact solutions. The exact solutions of Einstein's field equations are known as Lorentzian metrics obtained by solving these equations using the definition of energy-momentum tensor. Usually, there are two complementary approaches to get these exact solutions. For the first approach, one chooses a specific energy-momentum tensor model and studies the exact solutions corresponding to Einstein's field equations while assuming some physically acceptable properties on the scale factors. In the second approach, one focuses on some geometrical properties that are admitted by a space-time given by symmetries so as to simplify Einstein's field equations, and then searches for the matter source that depicts these properties. For the present investigation, we focus on a second approach, by assuming that a given space-time admits homothetic symmetry.

In the present work, we will focus our attention on the study of the homothetic symmetry of a Kantowski-Sachs space-time and solve Einstein's field equations without making assumptions, either on variables or on physical properties, as is common in the literature. We will only assume that a Kantowski-Sachs space-time understudy admits a homothetic vector.

Let $(M, g)$ be a space-time, that is, a smooth, 4-dimensional para-compact manifold $M$ with smooth metric $g$ of Lorentzian signature (+,-,-,-).

A global vector field $\zeta$ on $M$ is said to be homothetic if in any coordinate domain of $M$ one has

$$
£_{\zeta} g_{a b}=\zeta_{a ; b}+\zeta_{b ; a}=2 \psi g_{a b} \Leftrightarrow \zeta_{a ; b}=\psi g_{a b}+F_{a b},
$$

where $£$ denotes a Lie derivative and semi-colon denotes a covariant derivative with respect to metric connection. The vector field $\zeta$ is said to be homothetic if $\psi$ is constant on $M$ (proper homothetic vector field if $\psi \neq 0$ on $M$ ). If $\psi=0$ on $M$, the vector field $\zeta$ is said to be a Killing vector field on $M$.

The paper is organized as follows. In the next section, we will give a brief overview of the space-time understudy and obtain its physical and geometric properties and obtain the homothetic vector field that this space-time admits. Section 3 deals with Einstein's field equations of the model. Section 4 discusses the solutions of Einstein's field equations and their classifications of the space-times under consideration. In this Section 5, we give the kinematic quantities of the obtained solution. Section 6 deals with barotropic equation of state. Finally, in 
Section 7 concluding remarks are given.

\section{Version of Model and Homothetic Vector Field}

Consider a Kantowski-Sachs space-time which is a general measure of a homogeneous, anisotropic space-time with a spatial section of the topology $\mathfrak{R} \times S^{2}$. The standard representation of this space-time was given by [31]

$$
\mathrm{d} s^{2}=\mathrm{d} t^{2}-\mathrm{e}^{\Omega} \mathrm{d} r^{2}-R^{2}\left(\mathrm{~d} \theta^{2}+\sin ^{2} \theta \mathrm{d} \phi^{2}\right)
$$

with the convention $x^{0}=t$ (cosmic time), $x^{1}=r$ (transverse direction), $x^{2}=\theta$ and $x^{3}=\phi \quad$ (two equivalent longitudinal directions) and the scale factors $\Omega$ and $R$ are functions of $t$ only.

From the geometrical point of view, the line element (2.1) admits a four-parameter continuous group of isometries that acts on space-like hypersurface and has no three-parameter subgroup that would be simply transitive on the orbits (see the references [31] and [32]). The energy-momentum distribution of this space-time has been studied in [33] [34] [35].

In the following, we define the physical and geometric parameters for use in discussing the physical and geometric properties of the self-similar solution obtained from the metric (2.1).

The average scale factor $\tau$ of the Kantowski-Sachs model (2.1) is given by

$$
\tau=\left(\mathrm{e}^{\frac{\Omega}{2}} R^{2} \sin \theta\right)^{\frac{1}{3}},
$$

and $V$ represents a volume scale factor is defined as follows

$$
V=\tau^{3}=\mathrm{e}^{\frac{\Omega}{2}} R^{2} \sin \theta
$$

In view of the metric (2.1), according to Raychaudhuri [36], the 4-acceleration vector, rotation, expansion scalar and shear scalar characterizing the 4-velocity vector field, $u=\frac{\partial}{\partial t}$ with components $u^{a}=\delta_{0}^{a}$ can be written in a comoving coordinates system as follows

$$
\begin{aligned}
& \dot{u}_{a}=u_{a ; b} u^{b}=0, \\
& \Theta=u_{; a}^{a}=\frac{\dot{\Omega}}{2}+\frac{2 \dot{R}}{R}, \\
& \omega_{a b}=u_{[a ; b]}+\dot{u}_{[a} u_{b]}=0, \\
& \sigma^{2}=\frac{1}{18}\left(\frac{5 \dot{\Omega}^{2}}{2}+\frac{2 \dot{\Omega} \dot{R}}{R}+\frac{24 \dot{R}^{2}}{R^{2}}\right),
\end{aligned}
$$

where

$$
\sigma_{a b}=u_{(a ; b)}+\dot{u}_{(a} u_{a)}-\frac{1}{3} \Theta\left(g_{a b}+u_{a} u_{b}\right)
$$

The mean Hubbles parameter $H$ is defined as follows

$$
H=\frac{1}{3}\left(H_{r}+H_{\theta}+H_{\phi}\right) \text {, }
$$


where

$$
H_{r}=\frac{\dot{\Omega}}{2}, \quad H_{\theta}=H_{\phi}=\frac{\dot{R}}{R},
$$

are the directional Hubble's parameters which measure the rate of expansion in the directions of $r, \theta$ and $\phi$ respectively. A dot denotes a derivative with respect to cosmic time $t$. From Equations (2.2)-(2.5), we obtain

$$
H=\frac{\dot{\tau}}{\tau}=\frac{1}{3} \frac{\dot{V}}{V}=\frac{1}{3}\left(\frac{\dot{\Omega}}{2}+\frac{2 \dot{R}}{R}\right) .
$$

The deceleration parameter $q$ of a Kantowski-Sachs model is an important observational quantity, which is given as follows

$$
q=-\frac{\tau \ddot{\tau}}{\dot{\tau}^{2}}=\frac{\mathrm{d}}{\mathrm{d} t}\left(\frac{1}{H}\right)-1=-\frac{R^{2} \dot{\Omega}^{2}+8 R \dot{R} \dot{\Omega}-8 \dot{R}^{2}+6 R^{2} \ddot{\Omega}+24 R \ddot{R}}{(R \dot{\Omega}+4 \dot{R})^{2}}
$$

The anisotropic parameter of expansion $\delta$ is defined by

$$
\delta=\frac{1}{3} \sum_{i=1}^{3}\left(\frac{H_{i}}{H}-1\right)^{2}=\frac{1}{3} \sum_{i=1}^{3}\left(\frac{H_{i}^{2}}{H^{2}}-\frac{2 H_{i}}{H}+1\right),
$$

in which $H_{i}, i=1,2,3$ represent the directional Hubble's parameters as defined before.

For the space-time under consideration (2.1), the anisotropy parameter of the expansion is given by

$$
\delta=\frac{4}{3} \frac{R^{2} \dot{\Omega}^{2}-2 \dot{R}^{2}-10 R \dot{R} \dot{\Omega}}{(R \dot{\Omega}+4 \dot{R})^{2}} .
$$

Study of homothetic vector fields, $\zeta=\zeta^{a}(t, r, \theta, \phi)_{a=0}^{3}$, on Kantowski-Sachs model (2.1) is based on the examination of the ten equations obtained from the first equation of (1.1). For the model (2.1), the homothetic equations (1.1) are reduced to the following system of equations:

$$
\begin{gathered}
\zeta_{, 0}^{0}=\psi, \\
\zeta_{, 1}^{0}-\mathrm{e}^{\Omega} \zeta_{, 0}^{1}=0, \\
\zeta_{, 2}^{0}-R^{2} \zeta_{, 0}^{2}=0, \\
\zeta_{, 3}^{0}-R^{2} \sin ^{2} \theta \zeta_{, 0}^{3}=0, \\
\zeta_{, 1}^{1}+\left(\frac{\dot{\Omega}}{2}\right) \zeta^{0}=\psi, \\
e^{\Omega} \zeta_{, 2}^{1}+R^{2} \zeta_{, 1}^{2}=0, \\
\mathrm{e}^{\Omega} \zeta_{, 3}^{1}+R^{2} \sin ^{2} \theta \zeta_{, 1}^{3}=0, \\
\zeta_{, 2}^{2}+\left(\frac{\dot{R}}{R}\right) \zeta^{0}=\psi, \\
\zeta_{, 3}^{2}+\sin ^{2} \theta \zeta_{, 2}^{3}=0,
\end{gathered}
$$




$$
\zeta_{, 3}^{3}+\frac{\dot{R}}{R} \zeta^{0}+\cot \theta \zeta^{2}=\psi .
$$

Integrating (2.11) with respect to $t$ and using the obtained result back into (2.12), (2.13) and (2.14), after some algebraic calculations, we obtain

$$
\begin{aligned}
& \zeta^{0}=\psi t+c_{0}, \\
& \zeta^{1}=F_{1}(r, \theta, \phi), \\
& \zeta^{2}=F_{2}(r, \theta, \phi), \\
& \zeta^{3}=F_{3}(r, \theta, \phi),
\end{aligned}
$$

where $c_{0}$ is a constant of integration and $F_{1}(r, \theta, \phi), F_{2}(r, \theta, \phi)$ and $F_{3}(r, \theta, \phi)$ are arbitrary functions that are to be determined.

Differentiating Equations (2.15) and (2.18) with respect to $t$ and using (2.11) and (2.21), we get respectively

$$
\begin{aligned}
& \frac{\dot{\Omega}}{2}=\frac{a}{\psi t+c_{0}}, \\
& \frac{\dot{R}}{R}=\frac{b}{\psi t+c_{0}},
\end{aligned}
$$

where $a$ and $b$ are non-vanishing constants of integration. Substituting these results back into Equation (2.15) and Equation (2.18), using Equations (2.16), (2.17) and (2.19), we get

$$
\begin{gathered}
\zeta^{1}=(\psi-a) r+c_{1}, \\
\zeta^{2}=(\psi-b) \theta+c_{2},
\end{gathered}
$$

where $c_{1}$ and $c_{2}$ are constants of integration.

Using the previous results, we find from Equations (2.17) and (2.19) that $\zeta^{3}$ dos not depend on the variables $r$ and $\theta$. Consequently, Equation (2.20) leads to $\theta=\frac{\pi}{4}$ or $\psi=b$, and $c_{2}=0$; in both two cases $\zeta^{3}$ must equal constant. Since, we discuss the space-time under consideration for all values of $\theta$, therefore we choose the second case

$$
b=\psi \text {. }
$$

Hence

$$
\zeta^{3}=c_{3},
$$

where $c_{3}$ is a constant of integrations.

Without loss of generality, we assume that $c_{1}=c_{2}=c_{3}=0$, therefore, from Equations (2.21), (2.25), (2.26) and (2.28), we obtain the following homothetic vector field

$$
\zeta=\left(\psi t+c_{0}\right) \partial_{t}+(\psi-a) r \partial_{r} .
$$

From Equations (2.23) and (2.24), using (2.27), we get 


$$
\begin{aligned}
& \dot{\Omega}=\frac{2 a}{\psi t+c_{0}}, \\
& \frac{\dot{R}}{R}=\frac{\psi}{\psi t+c_{0}},
\end{aligned}
$$

Integrating the previous equation, we get the following self-similar solution for the metric (2.1) as follows (We took the constants of integration by unity)

$$
\begin{aligned}
& \Omega=\ln \left(\psi t+c_{0}\right)^{\frac{2 a}{\psi}}, \\
& R=\psi t+c_{0} .
\end{aligned}
$$

Our aim in the next section is to use the exact self-similar solution (2.31) in Einstein's field equations to obtain the dynamical variables.

\section{Einstein's Field Equations in the Case of a Perfect Fluid}

In this section, we will solve Einstein's field equations by assuming that the space-time under study admits a homothetic vector field and considering the matter is described by a perfect fluid.

Einstein's field equations are given by

$$
R_{a b}-\frac{1}{2} R g_{a b}=-T_{a b},
$$

(For convenience, we assumed that natural units $c=8 \pi G=1, G$ is the Newton's gravitational constant and $c$ is the speed of light in the vacuum), where $R_{a b}$ is the Ricci tensor, $R$ is the Ricci scalar and $T_{a b}$ is the energy momentum tensor that describes the physical ingredients of the space-time. In the case of a perfect fluid $T_{a b}$ is given by

$$
T_{a b}=(\rho+p) u_{a} u_{b}-p g_{a b},
$$

where $p$ is the pressure, $\rho$ the energy density and $u_{a}$ the four velocity vector, it must verify $£_{\zeta_{a}} u_{a}=0$. For the space-time (2.1), the contravariant and covariant components of the 4 -velocity vector can be defined by $u^{a}=u_{a}=(1,0,0,0)$ and these are verified $g_{a b} u^{a} u^{b}=1$.

For the line element (2.1), Einstein's field Equations (3.1) with (3.2) give the following system of equations

$$
\begin{gathered}
\frac{\dot{R} \dot{\Omega}}{R}+\frac{\dot{R}^{2}}{R^{2}}+\frac{1}{R^{2}}=\rho \\
\frac{2 \ddot{R}}{R}+\frac{\dot{R}^{2}}{R^{2}}+\frac{1}{R^{2}}=-p \\
\frac{\ddot{R}}{R}+\frac{\ddot{\Omega}}{2}+\frac{\dot{R} \dot{\Omega}}{2 R}+\frac{\dot{\Omega}^{2}}{4}=-p
\end{gathered}
$$

From the equation of stress-energy conservation $T_{a ; b}^{b}=0$, we get

$$
\dot{\rho}+(\rho+p)\left(\frac{\dot{\Omega}}{2}+2 \frac{\dot{R}}{R}\right)=0
$$




\section{Solutions of Einstein's Field Equations}

In this section, we'll solve Einstein's field equations for the space-time under study without making any assumption. We'll just assume that the space-time admits a homothetic vector. Using the scale factors (2.31) deduced from the condition that the space-time admits a homothetic vector. Equations (3.3)-(3.5) can be written as follows:

Equation (3.3) gives

$$
\rho=\frac{\psi^{2}+2 a \psi+1}{\left(\psi t+c_{0}\right)^{2}},
$$

and Equations (3.4) and (3.5) give, respectively

$$
\begin{aligned}
& p=-\frac{1+\psi^{2}}{\left(\psi t+c_{0}\right)^{2}}, \\
& p=-\frac{a^{2}}{\left(\psi t+c_{0}\right)^{2}} .
\end{aligned}
$$

From Equations (4.8) and (4.9), we obtain

$$
\psi^{2}+1=a^{2} .
$$

Using this relation in (4.7), we get

$$
\rho=\frac{\psi^{2}+2 \psi \sqrt{\psi^{2}+1}+1}{\left(\psi t+c_{0}\right)^{2}} .
$$

Equations (4.8) (or (4.9)) and (4.11) give the dynamical variables of the obtained self-similar solution (2.31). In the next section, we give its kinematic variables.

\section{Kinematic Variables}

In this section we give the kinematic quantities of the obtained solution (2.31) as follows:

The average scale factor $\tau$ and the volume scale factor $V$ are given, respectively, by

$$
\begin{gathered}
\tau=\left(\left(\psi t+c_{0}\right)^{\frac{2\left(\sqrt{\psi^{2}+1}+\psi\right)}{\psi}} \sin \theta\right)^{\frac{1}{3}}, \\
V=\tau^{3}=\left(\psi t+c_{0}\right)^{\frac{2\left(\sqrt{\psi^{2}+1}+\psi\right)}{\psi}} \sin \theta .
\end{gathered}
$$

In view of the solution (2.31), the 4 -acceleration vector, rotation, expansion scalar and shear scalar can be written, respectively, in a comoving coordinates system as follows

$$
\begin{aligned}
& \dot{u}_{a}=0, \\
& \Theta=\frac{\sqrt{\psi^{2}+1}+2 \psi}{\psi t+c_{0}},
\end{aligned}
$$




$$
\begin{aligned}
& \omega_{a b}=0, \\
& \sigma^{2}=\frac{5\left(\psi^{2}+1\right)+2 \psi \sqrt{\psi^{2}+1}+12 \psi^{2}}{9\left(\psi t+c_{0}\right)^{2}} .
\end{aligned}
$$

The directional and mean Hubbles parameters are

$$
\begin{gathered}
H_{r}=\frac{\sqrt{\psi^{2}+1}}{\psi t+c_{0}}, \quad H_{\theta}=H_{\phi}=\frac{\psi}{\psi t+c_{0}}, \\
H=\frac{2 \psi+\sqrt{\psi^{2}+1}}{3 \psi t+c_{0}}
\end{gathered}
$$

The deceleration parameter $q$ and the anisotropic parameter of expansion $\delta$ of the model (2.31) are given, respectively, as

$$
\begin{gathered}
q=-\frac{1-\psi^{2}+\psi \sqrt{\psi^{2}+1}}{\left(\sqrt{\psi^{2}+1}+2 \psi\right)^{2}}, \\
\delta=\frac{2\left(\psi^{2}-10 \psi \sqrt{\psi^{2}+1}+2\right)}{3\left(\sqrt{\psi^{2}+1}+2 \psi\right)^{2}}
\end{gathered}
$$

\section{Barotropic Equation of State}

As indicated in the references [8] [9] and [10] that if the matter is described by a perfect fluid, then the only barotropic equation of the state compatible with self-similarity is

$$
p=(\lambda-1) \rho
$$

where $\rho$ is the energy density, $p$ is the pressure and $\lambda$ is a constant in the range $1 \leq \lambda \leq 2$. However, this equation of state is physically consistent in the whole range of $\lambda$. When $\lambda=1$, the Equation (6.19) describes dust, $\lambda=4 / 3$ gives the equation of state for radiation and $\lambda=2$ considers the effective "stiff fluid" distribution. It would have been applied in the early Universe, because in this case, the speed of sound equals the speed of light, so there is no matter in this Universe could be stiffer.

From Equations (4.8) and (4.11), we get each of $p$ and $\rho$ depends on $\psi$. For comparison with the equation of state (6.19), we put

$$
\frac{p}{\rho}=-\frac{1+\psi^{2}}{\psi^{2}+2 \psi \sqrt{\psi^{2}+1}+1}=\lambda-1 .
$$

Now, we will study the following cases:

Case I: Dust case, $\lambda=1$

From Equation (6.20), we get $\psi^{2}=-1$, which gives imaginary values of $\psi$. Then the equation of state (6.19) does not satisfy in this case.

Case II: Radiation case, $\lambda=\frac{4}{3}$ 
In this case we obtain imaginary values of $\psi$, where $\psi=-\frac{4}{3}$. As in the previous case, the equation of stat (6.19) does not also satisfy.

Case III: Stiff fluid case, $\lambda=2$

In this case Equation (6.20) leads to an impossible equation of $\psi$. Consequently, the barotropic equation of state (6.19) does not also satisfy.

From the above discussion we obtained new classes of perfect fluid solutions whose matter energy density $\rho$ and pressure $p$ do not satisfy the barotropic equation of state (6.19).

\section{Discussion and Conclusion}

This work is devoted to studying the symmetries in particular self-similar symmetry of a Kantowski-Sachs model in the framework of Riemannian geometry. We have focussed on this kind of symmetry since space-time admitting it is stable from the dynamical system point and therefore is important from the physical one. For a Kantowski-Sachs space-time, we have solved the homothetic equations and found the homothetic vector field that this space-time admits. Moreover, the solution of homothetic equations helped us to get scale factors. We have used these scale factors in Einstein's field equations and found the dynamical variables, the energy density $\rho$ and the pressure $p$, which depend on the cosmic time $t$. Using the values obtained for these variables, we found that the equation of state, which was indicated by Cahill and Taub [8] and Bicknell and Henriksen [9], was not satisfied in the three cases, namely, dust, radiation and stiff fluid. Therefore, the obtained self-similar solution can be considered as an addition to the rare perfect fluid solutions which do not satisfy any barotropic equation of state. We discussed the kinematical quantities of the obtained solution (2.31), we found that the model is not accelerated and expanding with time because its volume element increases as the time increases, which gives essentially empty universe for large time. The Hubble parameter, the scalar expansion and the shear scalar assume infinitely large values whereas with the growth of cosmic time they decrease to null values as $t \rightarrow \infty$. The behavior of the fluid is time-dependent and can be physically reasonable. For the obtained solution the limit of the ratio as $t \rightarrow \infty$ is $\lim _{t \rightarrow \infty}\left(\frac{\sigma}{\Theta}\right) \neq 0$, that is, the anisotropy of the model is maintained throughout.

\section{Conflicts of Interest}

The authors declare no conflicts of interest regarding the publication of this paper.

\section{References}

[1] Hall, G.S. (2004) Symmetries and Curvature Structure in General Relativity. World Scientific, Singapore. https://doi.org/10.1142/1729

[2] Sussman, R.A. (1991) Spherically Symmetric Solutions Admitting a Spacelike 
Self-Similar Motion. Journal of Mathematical Physics, 32, 223.

https://aip.scitation.org/doi/abs/10.1063/1.529121

https://doi.org/10.1063/1.529121

[3] Gad, R.M. (2002) On Spherically Symmetric Perfect-Fluid Solutions Admitting Conformal Motions. Il Nuovo Cimento B, 11.

https://www.researchgate.net/publication/253424122_On_spherically_symmetric_p erfect-fluid_solutions_admitting_conformal_motions

[4] Stephani, H., Kramer, D., MacCallum, M.A.H., Hoenselears, C. and Herlt, E. (2003) Exact Solutions of Einstein's Field Equations. Cambridge University Press, Cambridge. https://doi.org/10.1017/CBO9780511535185

[5] Hall, G.S. (1996) The Mathematical Study of Symmetry in General Relativity. Gravitation and Cosmology, 2, 270-276. https://elibrary.ru/item.asp?id=12947286

[6] Hall, G.S. (1998) Some Remarks on Symmetries and Transformation Groups in General Relativity. General Relativity and Gravitation, 30, 1099-1110.

https://link.springer.com/article/10.1023/A:1026604822781 https://doi.org/10.1023/A:1026604822781

[7] Henriksen, R.N. and Wesson P.S. (1978) Self-Similar Space-Times I: Three Solutions. Astrophysics and Space Science, 53, 429-444.

https://link.springer.com/article/10.1007/BF00645031 https://doi.org/10.1007/BF00645031

[8] Cahill, M.E. and Taub, A.H. (1971) Spherically Symmetric Similarity Solutions of the Einstein Field Equations for a Perfect Fluid. Communications in Mathematical Physics, 21, 1-40. https://link.springer.com/article/10.1007/BF01646482 https://doi.org/10.1007/BF01646482

[9] Bicknell, G.V. and Henriksen, R.N. (1978) Self-Similar Growth of Primordial Black Holes. I. Stiff Equation of State. The Astrophysical Journal, 219, 1043-1057. https://adsabs.harvard.edu/pdf/1978ApJ...219.1043B https://doi.org/10.1086/155869

[10] Ori, A. and Piran, T. (1990) Naked Singularities and Other Features of Self-Similar General-Relativistic Gravitational Collapse. Physical Review D, 42, 1068-1090.

https://journals.aps.org/prd/abstract/10.1103/PhysRevD.42.1068 https://doi.org/10.1103/PhysRevD.42.1068

[11] Eardley, D.M. (1974) Self-Similar Spacetimes: Geometry and Dynamics. Communications in Mathematical Physics, 37, 287-309.

https://link.springer.com/article/10.1007/BF01645943 https://doi.org/10.1007/BF01645943

[12] Carter, B. and Henriksen, R.N. (1991) A Systematic Approach to Self-Similarity in Newtonian Space-Time. Journal of Mathematical Physics, 32, 2580.

https://aip.scitation.org/doi/abs/10.1063/1.529103 https://doi.org/10.1063/1.529103

[13] Wainwright, J. (2000) Asymptotic Self-Similarity Breaking in Cosmology. General Relativity and Gravitation, 32, 1041-1054.

https://link.springer.com/article/10.1023/A:1001917610163 https://doi.org/10.1023/A:1001917610163

[14] Wesson, P.S. (1978) An Exact Solution to Einstein's Equations with a Stiff Equation of State. Journal of Mathematical Physics, 19, 2283.

https://aip.scitation.org/doi/abs/10.1063/1.523605 https://doi.org/10.1063/1.523605

[15] Collins, M.E. and Lang, J.M. (1987) A Class of Self-Similar Perfect-Fluid Space- 
times, and a Generalisation. Classical and Quantum Gravity, 4, 61.

https://iopscience.iop.org/article/10.1088/0264-9381/4/1/009

https://doi.org/10.1088/0264-9381/4/1/009

[16] Gad, R.M. and Hassan, M.M. (2003) On The Geometrical and Physical Properties of Spherically Symmetric Non-Static Space-Times: Self-Similarity. Il Nuovo Cimento, 118B, 759-765. https://www.sif.it/riviste/sif/ncb/econtents/2003/118/08/article/8

[17] Medina, V. (2021) A Self-Similar Solution of a Fluid with Spherical Distribution in General Relativity. Indian Journal of Physics.

https://link.springer.com/article/10.1007/s12648-020-01959-1 https://doi.org/10.1007/s12648-020-01959-1

[18] Gad, R.M. (2009) On Spherically Symmetric Non-Static Space-Times Admitting Homothetic Motions. Il Nuovo Cimento, 124B, 61-67. https://www.sif.it/riviste/sif/ncb/econtents/2009/124/01/article/5

[19] Shabbir, G. and Khan, S. (2010) Classification of Bianchi Type I Spacetimes According to Their Proper Teleparallel Homothetic Vector Fields in the Teleparallel Theory of Gravitation. Modern Physics Letters A, 25, 2145-2153.

https://www.worldscientific.com/doi/abs/10.1142/S0217732310033189 https://doi.org/10.1142/S0217732310031403

[20] Shabbir, G., Ali, A. and Khan, S. (2011) A Note on Teleparallel Killing Vector Fields in Bianchi Type VIII and IX Space-Times in Teleparallel Theory of Gravitation. Chinese Physics B, 20, Article ID: 070401.

http://cpb.iphy.ac.cn/EN/10.1088/1674-1056/20/7/070401

https://doi.org/10.1088/1674-1056/20/7/070401

[21] Gad, R.M. and Alofi, A.S. (2014) Homothetic Vector Field in Plane Symmetric Bianchi Type-I Cosmological Model in Lyra Geometry. Modern Physics Letters A, 29, Article ID: 1450116.

https://www.worldscientific.com/doi/abs/10.1142/S0217732314501168 https://doi.org/10.1142/S0217732314501168

[22] Gad, R.M. (2015) Homothetic Motion in a Bianchi Type-I Model in Lyra Geometry. International Journal of Theoretical Physics, 54, 2932-2941.

https://link.springer.com/article/10.1007/s10773-015-2528-z https://doi.org/10.1007/s10773-015-2528-Z

[23] Alofi, A.S. and Gad, R.M. (2015) Homothetic Vector Fields in a Specially Homogenous Bianchi Type-I Cosmological Model in Lyra Geometry. Canadian Journal of Physics, 93, 1397-1401. https://cdnsciencepub.com/doi/abs/10.1139/cjp-2014-0555 https://doi.org/10.1139/cjp-2014-0555

[24] Gad, R.M. and Al Mazrooei, A.E. (2016) On Axially Symmetric Space-Times Admitting Homothetic Vector Fields in Lyra's Geometry. Canadian Journal of Physics, 94, 1148-1152. https://cdnsciencepub.com/doi/10.1139/cjp-2016-0114 https://doi.org/10.1139/cjp-2016-0114

[25] Hsu, L. and Wainwright, J. (1986) Self-Similar Spatially Homogeneous Cosmologies: Orthogonal Perfect Fluid and Vacuum Solutions. Classical and Quantum Gravity, 3, 1105. https://iopscience.iop.org/article/10.1088/0264-9381/3/6/011/meta https://doi.org/10.1088/0264-9381/3/6/011

[26] Carr, B.J. and Coley, A.A. (1999) Self-Similarity in General Relativity. Classical and Quantum Gravity, 16, R31.

https://iopscience.iop.org/article/10.1088/0264-9381/16/7/201

https://doi.org/10.1088/0264-9381/16/7/201

[27] Carr, B.J. and Gundlach, C. (2003) Spacetime Structure of Self-Similar Spherically 
Symmetric Perfect Fluid Solutions. Physical Review D, 67, Article ID: 024035. https://journals.aps.org/prd/abstract/10.1103/PhysRevD.67.024035 https://doi.org/10.1103/PhysRevD.67.024035

[28] Choptuik, M.W. (1993) Universality and Scaling in Gravitational Collapse of a Massless Scalar Field. Physical Review Letters, 70, 9-12.

https://journals.aps.org/prl/abstract/10.1103/PhysRevLett.70.9 https://doi.org/10.1103/PhysRevLett.70.9

[29] Gundlach, C. (2003) Critical Phenomena in Gravitational Collapse. Physics Reports, 376, 339-405.

https://www.sciencedirect.com/science/article/abs/pii/S0370157302005604 https://doi.org/10.1016/S0370-1573(02)00560-4

[30] Carr, B.J. and Coley, A.A. (2005) The Similarity Hypothesis in General Relativity. General Relativity and Gravitation, 37, 2165-2188.

https://link.springer.com/article/10.1007/s10714-005-0196-7

https://doi.org/10.1007/s10714-005-0196-7

[31] Kantowski, R. and Sachs, R.K. (1966) Some Spatially Homogeneous Anisotropic Relativistic Cosmological Models. Journal of Mathematical Physics, 7, 443.

https://doi.org/10.1063/1.1704952

[32] Collins, C.B. (1977) Global Structure of the "Kantowski-Sachs" Cosmological Models. Journal of Mathematical Physics, 18, 2116.

https://aip.scitation.org/doi/abs/10.1063/1.523191 https://doi.org/10.1063/1.523191

[33] Gad, R.M. and Fouad, A. (2007) Energy and Momentum Distributions of Kantowski and Sachs Space-Time. Astrophysics and Space Science, 310, 135-140. https://link.springer.com/content/pdf/10.1007/s10509-007-9488-6.pdf https://doi.org/10.1007/s10509-007-9488-6

[34] Abdel-Megied, M. and Gad, R.M. (2010) Möller's Energy in the Kantowski-Sachs Space-Time. Advances in High Energy Physics, 2010, Article ID: 379473. https://www.hindawi.com/journals/ahep/2010/379473 https://doi.org/10.1155/2010/379473

[35] Gad, R.M. and Mourad, M.F. (2008) Energy Distributions of Kantowski-Sachs Space-Time in the Theory of Teleparallel Gravity. Astrophysics and Space Science, 314, 341-346. https://link.springer.com/article/10.1007/s10509-008-9775-x https://doi.org/10.1007/s10509-008-9775-x

[36] Raychaudhuri, A.K. (1979) Theoretical Cosmology. Clarendon, Oxford. 